\begin{tabular}{|c|c|}
\hline Title & $\begin{array}{l}\text { A } n \text { experimental validation of a detailed numerical model for predicting implantable medical devices EMI due to low- } \\
\text { band RFID reader/writers }\end{array}$ \\
\hline Author(s) & $\begin{array}{l}\text { Futatsumori, Shunichi; Toyama, Norihiro; Hikage, Takashi; Nojima, Toshio; Koike, Ben; Fujimoto, Hiroshi; } \\
\text { T oy oshima, Takeshi }\end{array}$ \\
\hline Citation & $\begin{array}{l}\text { ISBN: 978-1-4244-2641-6, 1-4 } \\
\text { https://doi.org/10.1109/APMC.2008.4958016 }\end{array}$ \\
\hline Issue Date & $2008-12-16$ \\
\hline Doc URL & http:/hdl.handle.net/2115/38988 \\
\hline Rights & $\begin{array}{l}\text { C2008 IEEE. Personal use of this material is permitted. However, permission to reprint/republish this material for } \\
\text { advertising or promotional purposes or for creating new collective works for resale or redistribution to servers or lists, } \\
\text { or to reuse any copyrighted component of this work in other works must be obtained from the IEEE. }\end{array}$ \\
\hline Type & proceedings \\
\hline Note & A PMC 2008 (A sia Pacific Microwave Conference, 2008). 16-20 Dec. 2008. Macau \\
\hline File Information & A PMC2008_p1-4.pdf \\
\hline
\end{tabular}

Instructions for use 


\section{An Experimental Validation of a Detailed Numerical Model for Predicting Implantable Medical Devices EMI Due to Low-Band RFID reader/writers}

Shunichi Futatsumori ${ }^{1}$, Norihiro Toyama ${ }^{1}$, Takashi Hikage ${ }^{1}$, Toshio Nojima ${ }^{1}$, Ben Koike ${ }^{2}$ Hiroshi Fujimoto ${ }^{3}$, and Takeshi Toyoshima ${ }^{3}$

${ }^{1}$ Graduate School of Information Science and Technology, Hokkaido University, Japan

${ }^{2}$ Japan Automatic Identification Association, Japan

${ }^{3}$ Medtronic Japan Co., Ltd., Japan

${ }^{1}$ futatsumori@emwtinfo.ice.eng.hokudai.ac.jp

\section{Introduction}

Wireless communication devices such as mobile phones, radio-frequency identification (RFID), electric article surveillance (EAS), and contactless IC cards are essential devices to realize the ubiquitous and universal network society. On the other hand, since they are wireless devices, they emit electromagnetic fields (EMF) that could potentially cause electromagnetic interference (EMI) for other electromagnetic devices. The EMI on implantable medical devices (IMDs) such as implantable-cardiac pacemakers and cardioverter defibrillators is one of the most important issues needing investigation [1], [2]. This is because the number of IMD users is increasing every year due to technological advances in IMDs as well as due to the aging of the population (more persons using IMDs).

In this paper, a numerical assessment methodology of EMI occurrence in IMD due to lowband RFID reader/writer (RFID/IMD-EMI) is confirmed based on the results of in-vitro experiments and FDTD analyses. First, the EMI is outlined using the operating principle of tag communication. Secondly, in-vitro EMI experiments using fabricated experimental RFID reader/writer antennas and implantable-cardiac pacemakers are discussed. Then, the maximum interference distance obtained by the experiments and the numerical analyses are compared in order to validate the assessment methodology.

\section{Implantable Medical Device EMI Due to Low-Band RFID Reader/writers}

The low-band RFID systems described in this paper are HF (13.56 MHz) RFID reader/writers. Since they provide electric power to passive tags via inductive coupling, the reader/writer antennas generate relatively strong magnetic fields. When the alternating magnetic field, which is generated by the RFID reader/writer antenna, passes through the antenna coil of the tag, an induction voltage is produced. This interaction follows Faraday's law of induction. The induced voltage $V_{i}$ is given by:

$$
V_{i}=-N \frac{d \Phi_{t}}{d t}
$$

where $\Phi_{t}$ is the magnetic flux through the area perpendicular to the coil, and $N$ is the number of turns of the tag coil. $\Phi_{t}$ is a scalar product of the magnetic flux density $\vec{B}$ and the area of the tag coil $\vec{S}$. The induced voltage is rectified to DC voltage and activates the tag's function. However, the sensing circuit of the IMD may suffer induced voltages yielding EMI. This is because the lead wire of the IMD and the tissues of human body are assumed to compose a "one turn coil". The induced voltage generated in the terminal of IMDs is defined as "interference voltage". If the interference voltage exceeds the IMD's sensing threshold level, it cannot recognize correct electrocardiogram (ECG) signals and may malfunction [3].

\section{In-vitro EMI Experiments Using Implantable-Cardiac Pacemakers}


To obtain EMI characteristics of actual IMDs, in-vitro experiments using 2 types of pacemakers and 4 types of experimental RFID reader/writer antenna are carried out. The parameter values of the two pacemakers are shown in Table 1. Figure 1 shows the measurement system used for the experiments. This test system is based on the one previously proposed for the estimation of EMI due to mobile phones described in [1], [4]. The test system consists of a flat human torso phantom, an ECG signal generator, a chart recorder, an oscilloscope, a measurement platform and an RFID reader/writer antenna. The human torso phantom shown in Fig. 2 is a modification of Irnich's model.

The 4 types of experimental RFID reader/writer antenna are composed of a copper coil element and a balanced capacitor matching circuit. The return loss (better than $-20 \mathrm{~dB}$ ) and the quality factor (less than 20) of fabricated antennas are adjusted to values consistent with actual commercially available antennas. In addition, to investigate the difference in EMI due to antenna coil size, the width and the height of antenna are varied. The dimensions of antenna coils (width $\times$ height) are $300 \mathrm{~mm} \times 300 \mathrm{~mm}$ (Antenna A), $350 \mathrm{~mm} \times 270 \mathrm{~mm}$ (Antenna B), $200 \mathrm{~mm} \times 200 \mathrm{~mm}$ (Antenna C), and $100 \mathrm{~mm} \times 100 \mathrm{~mm}$ (Antenna D). Figure 3 shows the layout of Antenna A. In addition, Fig. 4 shows the overview of the EMI test system. The torso phantom is positioned parallel to the antenna. The distance between antennas and the torso phantom is varied in order to determine the maximum interference distance (distance where EMI disappears). The maximum interference distance at the different antenna input powers are measured in centimeters.

\section{Estimation of EMI Occurrence Based on Numerical Analysis}

To validate the assessment methodology based on the numerical analysis, the maximum interference distance obtained by the experiments and the numerical analyses are compared. The torso phantom model and RFID reader/writer antennas are modeled and analyzed. Figure 5 shows the torso phantom model, which contains the pacemaker model and the antenna model. Dielectric constant and electric conductivity values at $13.56 \mathrm{MHz}$ are used in the calculations. In addition, the torso phantom model is set to be parallel to the antenna model, which is the same condition used in the experiments. The interference voltage is evaluated at the both ends of the resistance, which is attached between the metal housing of the pacemaker model and the inner conductor of coaxial lead wire model, as shown in Fig. 6.

The interference voltage generated by the 4 types of antennas is obtained by using the FDTD method. The analysis parameters are shown in Table 2. Figure 7 shows the interference voltage with respect to the distance between antennas and the torso phantom. Then, Fig. 8 shows an example of the maximum interference distance obtained by the experiments and the numerical analyses. The analyzed values for the 4 types of antennas agree well with the measured values. From these results, the fundamental validation of assessment methodology is confirmed.

\section{Conclusions}

The numerical assessment methodology of RFID/IMD-EMI was confirmed based on the result of the in-vitro experiments and the numerical analyses. The maximum interference distances obtained by the experiments and the FDTD analysis agreed very well. The good repeatability of the experiments and detailed modeling in the analyses achieve this high accuracy estimation. These results clarify the interference voltages due to the magnetic field generated around the low-band RFID reader/writer and they can be estimated by using precise and detailed analysis. 
Acknowledgements: The authors would like to thank the members of the Pacemaker Committee of Japan and Japan Automatic Identification Association for their cooperation and support.

\section{References}

[1] W. Irnich, L. Batz, R. Muller and R. Tobisch, "Electromagnetic interference of pacemakers by mobile phones," J. Pacing and Clinical Electrophysiology, vol. 19, no. 10, pp.1431-1446, Oct. 1996.

[2] Y. Tarusawa, K. Oshita, Y. Suzuki, T. Nojima and T. Toyoshima, "Experimental estimation of EMI from cellular base-station antennas on implantable cardiac pacemakers," IEEE Trans. Electromagn. Compat., vol. 47, no. 4, pp. 938-950, Nov. 2005.

[3] S. Futatsumori, T. Hikage, T. Nojima, B. Koike, H. Fujimoto and T. Toyoshima, "A novel assessment methodology for the EMI occurrence in implantable medical devices based upon magnetic flux distribution of RFID reader/writers," in Proc. 2007 IEEE Int. Symp. on Electromagnetic Compatibility, pp. 1-6, 2007.

[4] Active Implantable Medical Device - Electromagnetic Compatibility - EMC Test Protocols for Implantable Cardiac Pacemakers and Implantable Defibrillators, AAMI Standard PC69, 1999.

Table 1. Parameter values for the pacemakers

\begin{tabular}{cc}
\hline Parameter & Value \\
\hline Stimulation mode & AAI or VVI \\
Heart rate & $60 \mathrm{bpm}$ \\
Pacing/sensing polarity & Unipolar or bipolar \\
Pulse amplitude/duration & Nominal values \\
Sensitivity & Maximum \\
Refectory period & (most sensitive) \\
\hline
\end{tabular}

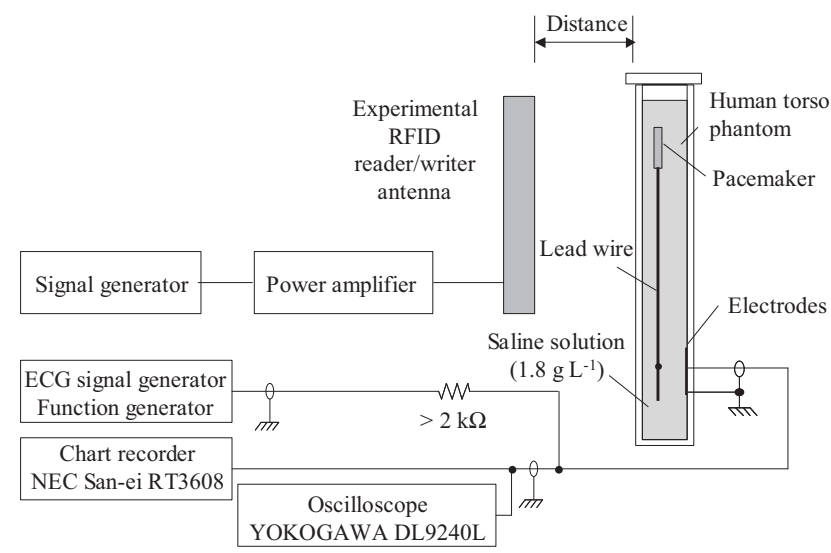

Figure 1. The configuration of the test system

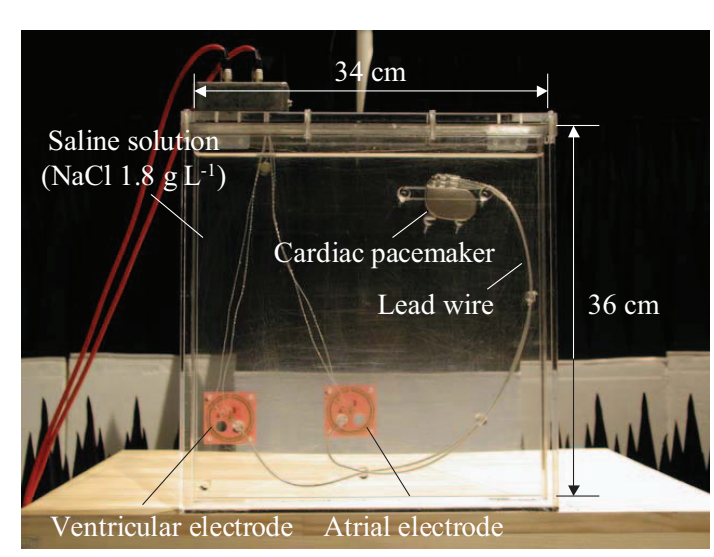

Figure 2. The torso phantom

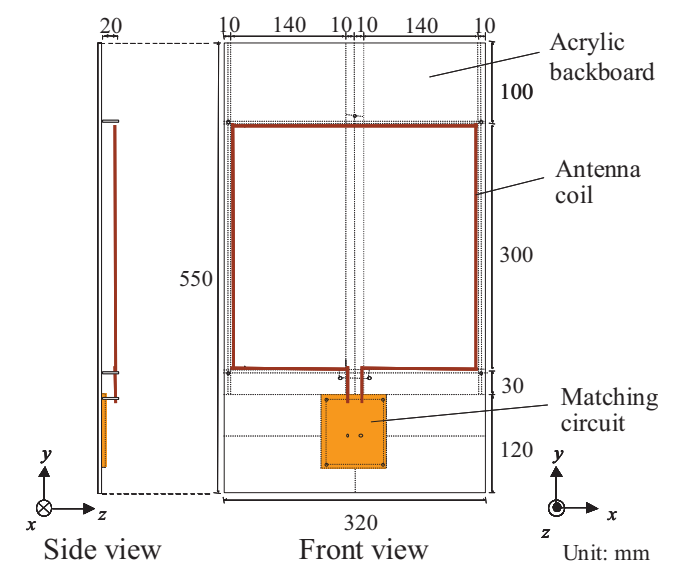

Figure 3. An example of experimental antenna layout 


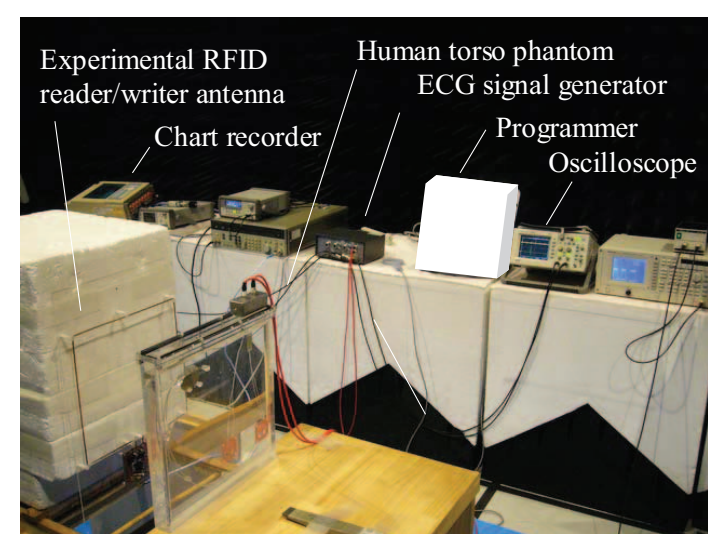

Figure 4. Overall view of the test system

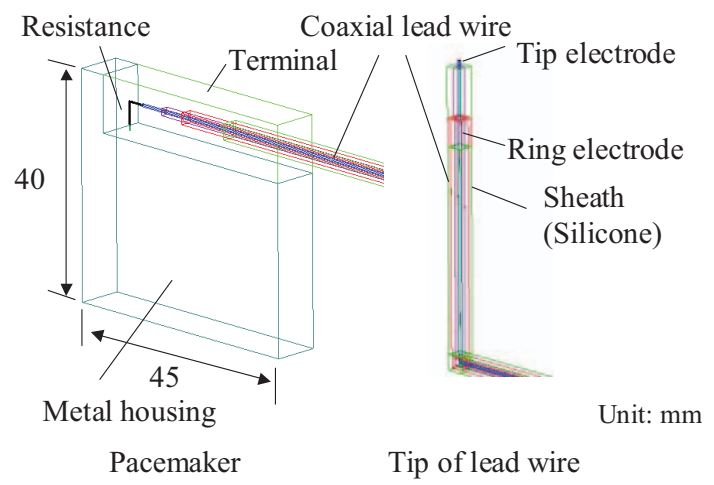

Figure 6. The numerical model of the pacemaker and the lead wire

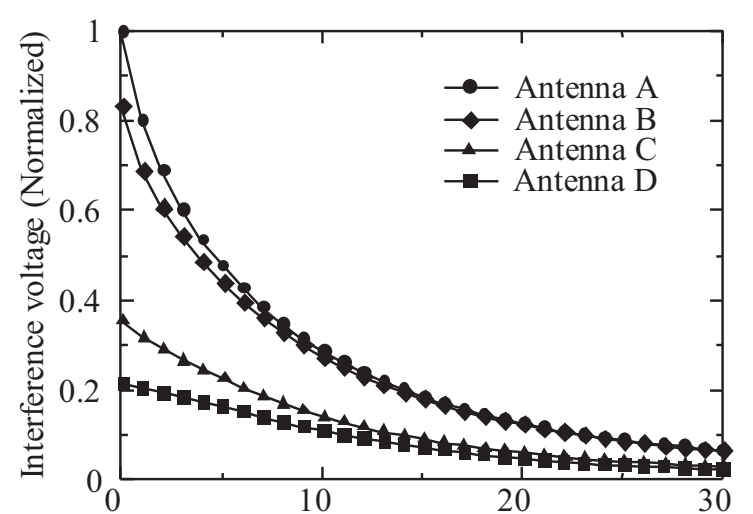

Distance from the surface of RFID reader/writer antenna $(\mathrm{cm})$

Figure 7. The interference voltage obtained by the analyses

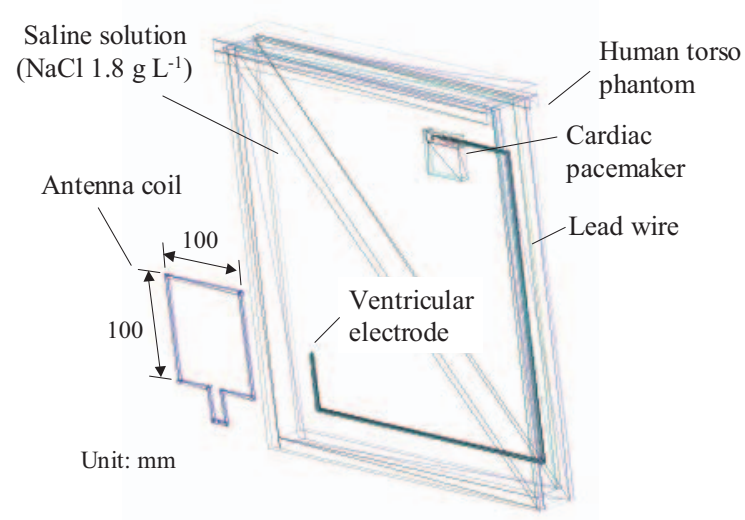

Figure 5. An example of the numerical models (Antenna D)

Table 2. The analysis parameters

\begin{tabular}{cc}
\hline Frequency & $13.56 \mathrm{MHz}$ \\
Cell size (minimum) & $\Delta=0.1 \mathrm{~mm}$ \\
Total problem space & $0.7-0.8 \mathrm{Mcells}$ \\
Absorbing B.C & PML $(8 \mathrm{Layer}, \mathrm{M}=4)$ \\
Period & $3-5$ periods \\
Feeding method & Delta gap \\
Antenna model & Coil: PEC \\
& NaCl solution: $\varepsilon_{\mathrm{r}}=86.7$, \\
& $\sigma_{\mathrm{E}}=0.32 \mathrm{~S} / \mathrm{m}$ \\
Phantom model & Acrylic tank: $\varepsilon_{\mathrm{r}}=3, \sigma_{\mathrm{E}}=0$ \\
& Cardiac pacemaker and \\
& lead wire: $\mathrm{PEC}$ \\
& Silicone: $\varepsilon_{\mathrm{r}}=2.7, \sigma_{\mathrm{E}}=0$ \\
\hline
\end{tabular}

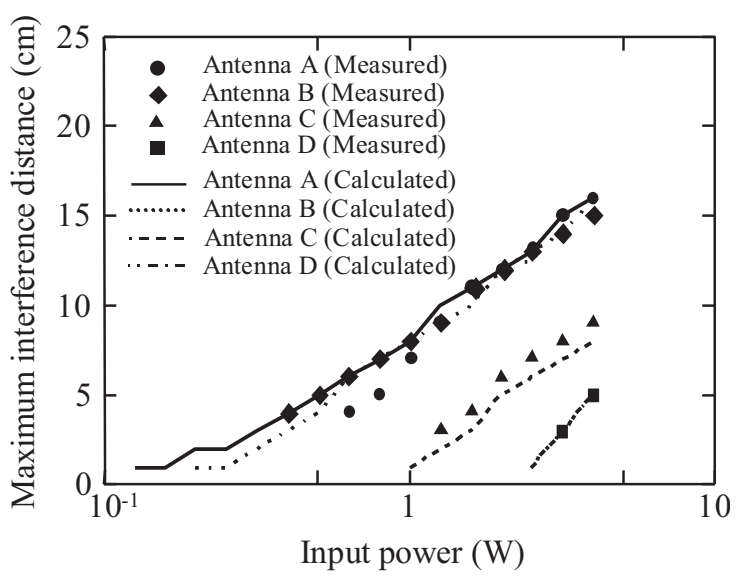

Figure 8. The measured and calculated maximum interference distance 